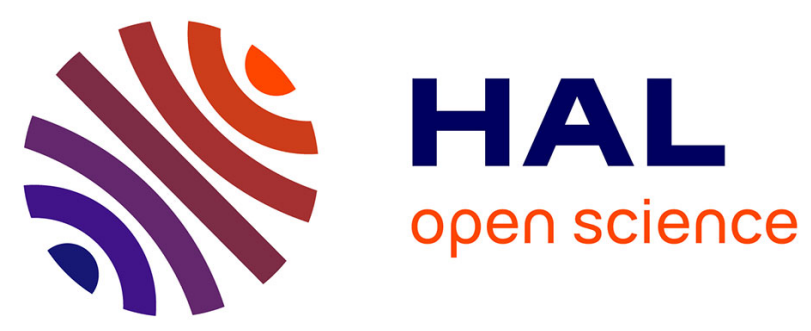

\title{
Accuracy Assessment of a Deep-Learning Based Segmentation Tool Over Right Ventricle Short-Axis Slices
}

\author{
Asma Ammari, Ramzi Mahmoudi, Badii Hmida, Rachida Saouli, Mohamed \\ Hédi Bedoui
}

\section{To cite this version:}

Asma Ammari, Ramzi Mahmoudi, Badii Hmida, Rachida Saouli, Mohamed Hédi Bedoui. Accuracy Assessment of a Deep-Learning Based Segmentation Tool Over Right Ventricle Short-Axis Slices. Digital Health in Focus of Predictive, Preventive and Personalised Medicine, pp.121-128, 2020, 10.1007/978-3-030-49815-3_14. hal-03112514

\section{HAL Id: hal-03112514 https://hal.science/hal-03112514}

Submitted on 16 Jan 2021

HAL is a multi-disciplinary open access archive for the deposit and dissemination of scientific research documents, whether they are published or not. The documents may come from teaching and research institutions in France or abroad, or from public or private research centers.
L'archive ouverte pluridisciplinaire HAL, est destinée au dépôt et à la diffusion de documents scientifiques de niveau recherche, publiés ou non, émanant des établissements d'enseignement et de recherche français ou étrangers, des laboratoires publics ou privés. 


\title{
Accuracy Assessment of a Deep-Learning Based Segmentation Tool Over Right Ventricle Short-axis Slices
}

\author{
Asma AMMARI ${ }^{1,2,3}$ [0000-0002-1989-621X], Ramzi MAHMOUDI ${ }^{2,4}$ [0000-0002-4271-3506] \\ Badii HMID ${ }^{5}$ [0000-0003-3109-1331], Rachida SAOULI ${ }^{3,4}$ [0000-0002-4299-2664], \\ Mohamed Hédi BEDOUI ${ }^{2}$ [0000-0003-4846-1722] \\ ${ }^{1}$ The National Engineering School L'ENIS, SFAX, TUNISIA \\ ${ }^{2}$ Faculty of Medicine of Monastir, Medical Imaging Technology Lab - LTIM-LR12ES06, Uni- \\ versity of Monastir - 5019 MONASTIR, TUNISIA. \\ ${ }^{3}$ Department of computer science, Laboratory of Intelligent Computing (LINFI), Mohamed \\ Khaider University BP 145 RP 07000, BISKRA, ALGERIA. \\ ${ }^{4}$ Gaspard-Monge computer-science laboratory, Paris-Est University, Mixed Unit CNRS-UMLV- \\ ESIEE UMR8049, BP99, ESIEE Paris City Descartes, 93162 Noisy Le Grand, FRANCE. \\ ${ }^{5}$ Radiology Service- UR12SP40 CHU Fattouma Bourguiba - 5019 MONASTIR, TUNISIA. \\ asma.ammari@enis.tn
}

\begin{abstract}
The right ventricle (RV) is the largest heart cavity playing a vital role in the cardiac cycle and function. However, to assess its function in cardiac magnetic resonance imaging (CMRI), the segmentation is an essential but very challenging task due to the RV complex crescent shape, thin boundaries, as well as the high variability of images. Indeed, to overcome these issues, several approaches have been proposed. In this paper, we aim to highlight the impact of various short-axis slices on the segmentation process. For that sake, based on the Cvi42 advanced deep learning base software, we assess the effectiveness of the $\mathrm{RV}$ contour delineation process over an entire MRI cardiac short-axis slice. We consider for evaluating the accuracy, the Dice Score Metric (DSM), as well as some RV functional parameters such as the Ejection Fraction, the End-SystolicVolume, and the End-Diastolic-Volume. Our experimental assessment is done using two CMRI exams of both healthy and diseased patients. We consider for evaluation the whole short-axis frames from base to apex and from end-systole to end-diastole. The evaluation results show that: (1) The segmentation accuracy is influenced by the slice level especially on the basal and apical slices, (2) A huge accuracy decreasing for the case of a patient having dysplasia.
\end{abstract}

Keywords: Right Ventricular, Cardiac segmentation tool, Endocardium, Functional parameters, Accuracy assessment.

\section{Introduction}

In the cardiac system, both ventricles have the role of pumping blood in the whole body. For the sake of enabling this organized role, the ventricular structure allows its function. Still, each ventricle has its own personalized morphology. Unlike the left ventricle $(\mathrm{LV})$, the right ventricle $(\mathrm{RV})$ has harsher trabeculae with a fibrous discontinuity 
between valves. The RV provides a thin wall and a crescent shape which makes it difficult to be modelled geometrically [1]. The assessment of the LV is widely handled using many segmentation techniques, even at the clinical practices, they segment and quantify its function automatically [2-4]. However, the RV is routinely assessed manually by expert radiologists to delineate its boundaries through the short axis several slices which are a fastidious, laborious, and time-consuming task. Therefore, automated assessment is crucial especially with the RV importance increasing for numerous pathological diagnosis. Irrefutably, its complex structure makes the segmentation task very challenging. Although many approaches have been proposed to handle those issues [5], still the problem of images variability and shape deformation from base to apex influence the accuracy of the proposed methods. In this paper, our goal is to study the impact of the RV MRI short-axis different slices on the segmentation accuracy using a commercially available cardiac MRI analysis software (CVi42, version 5.5.1, Circle Cardiovascular Imaging Inc., Calgary, Canada) [6]. Thus, we use two MRI exams of sick and healthy patients to experiment segmentation using the tool mentioned above. Based on the ground truth drawn by the expert, we compute the accuracy, the dice score metric, and other functional parameters related to the RV itself. The rest of this paper is organized as follow: We start by the state of the art in section 2 followed by a description of the used image data and the experimented platform in section 3. The experimental results are introduced in section 4. Finally, in section 5, discussion and conclusion are given.

\section{State of the art}

The existing attempts to overcome the challenges related to the RV segmentation make use of many medical imaging segmentation techniques [5]. Based on prior information, in $[7,8]$, multi-atlas based approaches are exploited. Since atlas-based methods depend at the first stage on the database used, its results may fail to provide high accuracy when the segmented cardiac images present different structures from those used in the first place. Deformable models are also used for RV segmentation such in [9] and [10]. These methods are based on the shape information of the right ventricular. On the other hand, other works make use of graph cuts-based methods hybridized with several imaging techniques aiming thus to segment the right ventricular boundaries [11], [12]. Recently with the appearance and the augmented use of deep learning techniques to solve several imaging problems. In [12], deep learning methods are used for right ventricular segmentation. These methods depend on used architecture to train the model as well on the amount and the variation of used training sets. In this paper, we attempt to highlight the main issues that might hold back the adaptation of the promising deeplearning solutions for the RV challenging case considering at the first place the RV contour variation from base to apex. For that interest, we investigate the Cvi42 segmentation results, as it is based on a deep-learning approach using a fully conventional neural network trained on thousands of CMRI subjects acquired from the UK Biobank. 


\section{Image data and Experiment Platform}

To allow the Cvi42 cardiac segmentation tool assessment, the image data used encompasses a short-axis cine cardiac MR DICOM images which belong to two patients gathered from Fattouma Bourguiba Monastir Hospital in Tunisia. One patient presents a normal case while the other suffers from dysplasia in which the RV surface appears dilated. The manual RV delineation for both patients' sequences performed by the expert, based on the 4-chambers and the long-axis slices, is shown in Fig. 1 which is to be used as a referential segmentation. The experiment is done using a 5.10 version of Cvi42 upon an i7 ASUS platform (2.4 GHz CPU, 8GB RAM, Nvidia GeForce 920 graphical card). The evaluation metrics are computed using MATLAB R2016a.
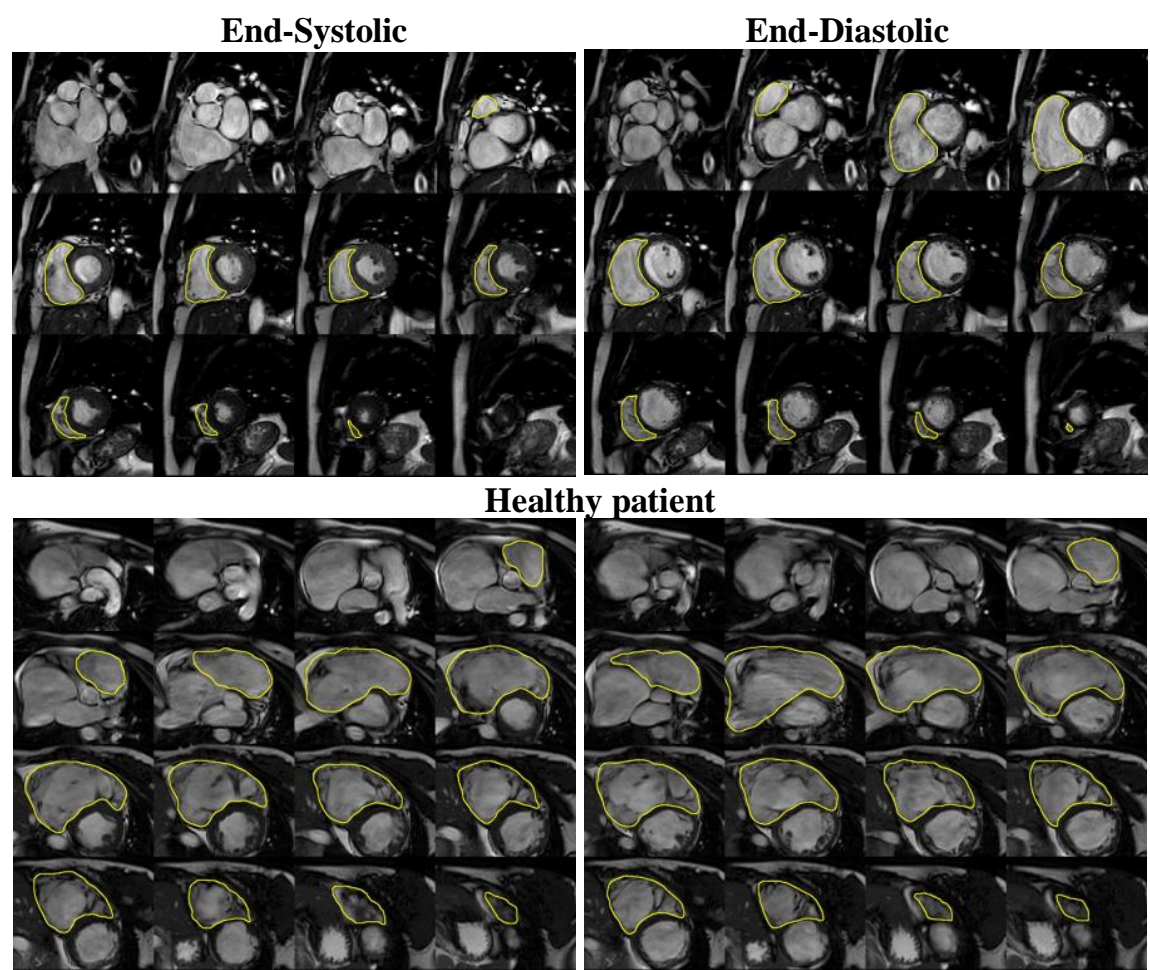

Dysplasia patient

Fig. 1. Expert's manual RV endocardium delineation in end-systole and end-diastole for both healthy and diseased patient. (For interpretation of segmentation yellow color in this figure, the reader is referred to the web version of this article)

\section{$4 \quad$ Experimental results}

In this section, the obtained RV boundaries automatic delineation, as well as the precession evaluation, are presented. 


\subsection{Segmentation results}

Fig. 2 shows the segmentation results of the automated segmentation tool, in which the yellow line is the result of endocardium delineation. Compared to manual segmentation, we notice that the software has failed to detect the first basal and the last apical slices in the case of the healthy patient. However, in the case of dysplasia patient, most of the apical slices were missed out as well as some basal slices. Furthermore, the precession of contouring is clearly over and underestimated especially for the diseased case. The exact metrical evaluation of these delineation results is assessed in the following sub-section.
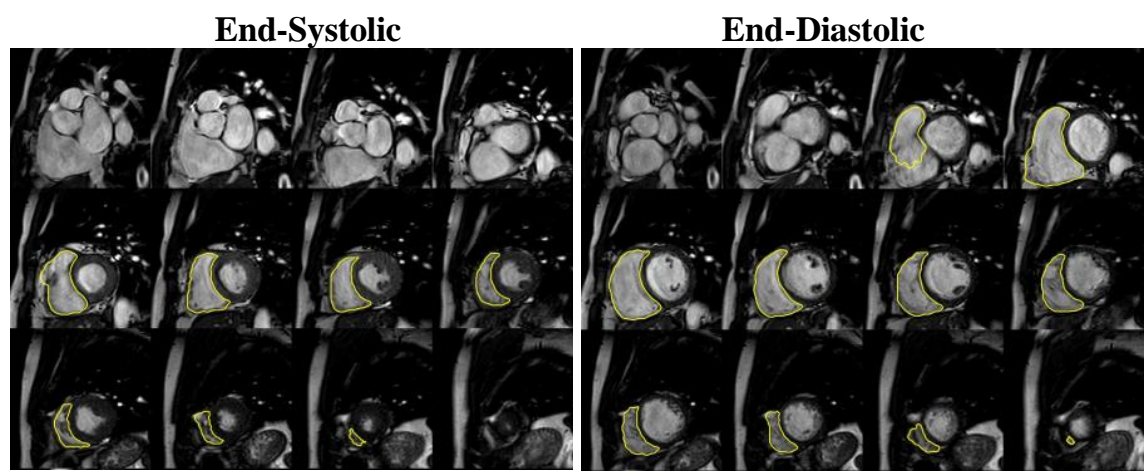

Healthy patient

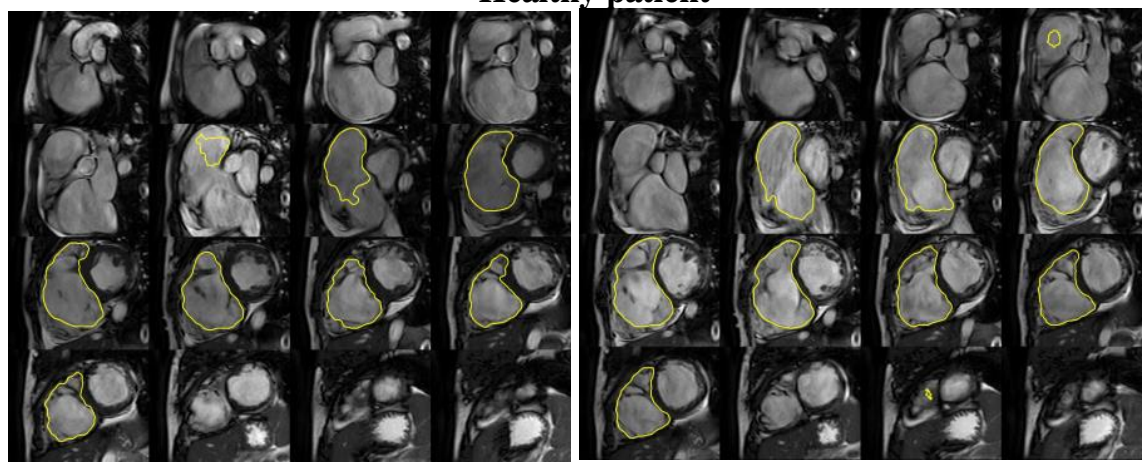

Dysplasia patient

Fig. 2. Automatic Cvi42 RV endocardium delineation in end-systole and end-diastole for both healthy and diseased patient. (For interpretation of segmentation yellow color in this figure, the reader is referred to the web version of this article)

\subsection{Empirical evaluation}

In this paper, we compute the Dice metric (DM) and the accuracy for the sake of evaluating the performance of the RV automatic segmentation offered by the tool (Cvi42). The DM is the overlap between the automatic segmentation result and the manual segmentation result computed following the equation (1) [5], and the accuracy computed 
based on true positives, true negatives, false positives and false negatives as it is described in the equation (2). Graphics in Fig. 3 and Fig4 show respectively the obtained results for the Accuracy and the DM.

$$
\begin{gathered}
\operatorname{DM}(U, V)=2 \frac{U V}{U+V} \\
\text { Accuracy }=\frac{T P+T N}{T P+T N+F P+F N}
\end{gathered}
$$

Table.1 shows some functional parameters such as RV End-systolic Volume, RV EndDiastolic Volume, and the ejection fraction of the assessed cases. The presented values obtained by the expert and the segmentation tool might be computed according to the equations $(3,4,5)$.

$$
\begin{gathered}
R V E S V=\min _{t}[V(t)] \\
R V E D V=\max _{t}[V(t) \\
R V E F(\%)=\frac{R V E D V-R V E S V}{R V E D V} \times 100
\end{gathered}
$$

Considering both the RV-end-systolic-volume and the RV-end-diastolic-volume, the values obtained by the segmentation tool seem to be higher compared to the values obtained by the expert for the case of the healthy patient. However, in the case of the diseased patient, we find that the automatically obtained volumes show lower values than those obtained by the expert. Consequently, the resulting ejection fraction obtained by the automatic segmentation is lower for the case of the healthy patient and higher for the diseased one compared to the expert' referential results. Indeed, as the main goal of the RV boundaries segmentation is to allow accurate functional parameters computing. Thus, any under or over-estimated contours even at the basal or apical slices might influence the obtained parametrical results especially for the case of pathological pa-

\begin{tabular}{|c|c|c|}
\hline & Healthy patient & Diseased patient \\
\hline Ground truth & $\begin{array}{l}\text { RVEDV : } 75.4 \mathrm{ml} / \mathrm{m}^{2} \\
\text { RVESV }: 32.2 \mathrm{ml} / \mathrm{m}^{2} \\
\text { RVEF }: 57 \%\end{array}$ & $\begin{array}{l}\text { RVEDV : } 491 \mathrm{ml} \\
\text { RVESV }: 410 \mathrm{ml} \\
\text { RVEF }: 6 \%\end{array}$ \\
\hline Automatic & $\begin{array}{l}\text { RVEDV }: 209.52 \mathrm{ml} \\
\text { RVESV }: 114.45 \mathrm{ml} \\
\text { RVEF }: 45.37 \%\end{array}$ & $\begin{array}{l}\text { RVEDV : } 401.04 \mathrm{ml} \\
\text { RVESV : } 276.12 \mathrm{ml} \\
\text { RVEF }: 31.15 \%\end{array}$ \\
\hline
\end{tabular}
tients where neither the size nor the end-systolic and the end-diastolic phases are normal.

Table 1. Right ventricular functional parameters. (RVESV): RV End Systolic Volume, (RVEDV): RV End Diastolic Volume, RVEF): RV Ejection Fraction. 


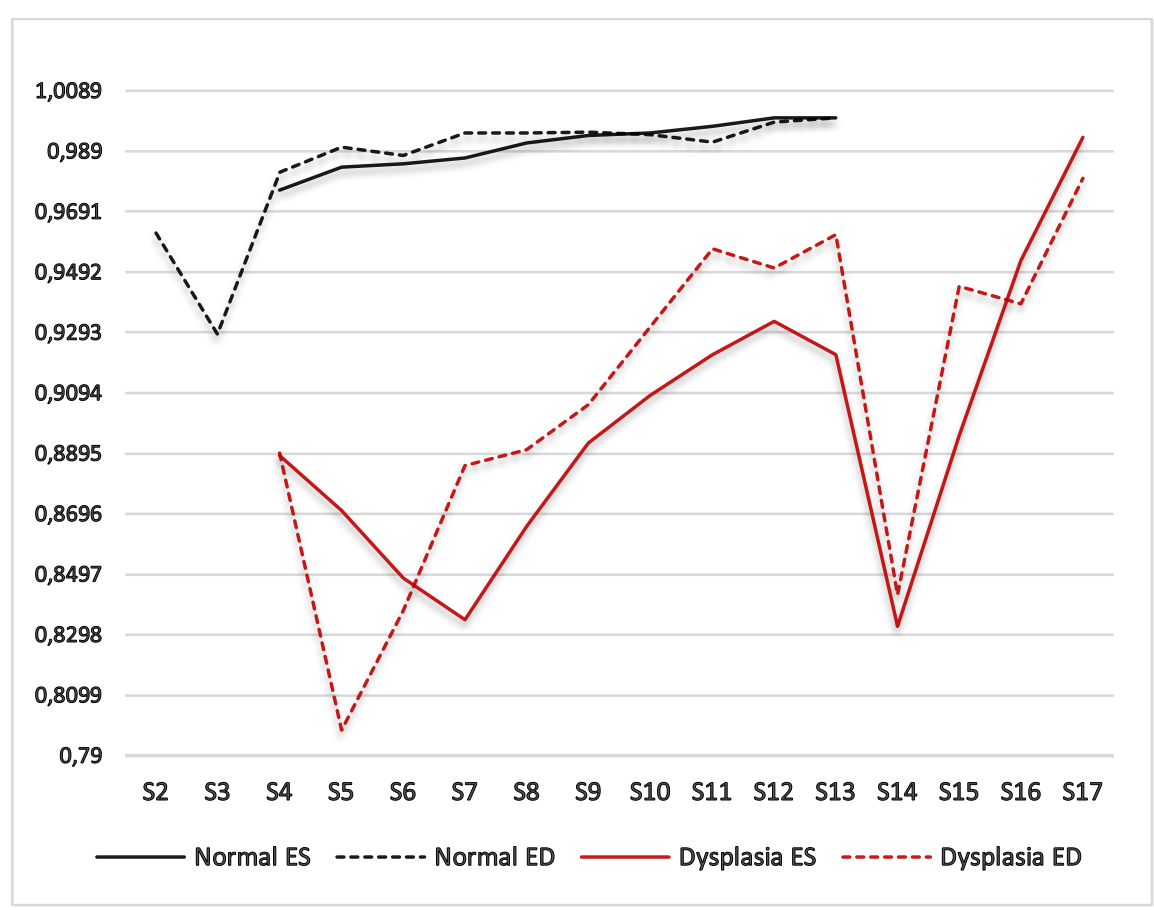

Fig. 3. Accuracy evaluation of Short-Axis Slices from $(S 2 \rightarrow S 17)$ for both End-Systolic and EndDiastolic phases considering both normal and diseased patients

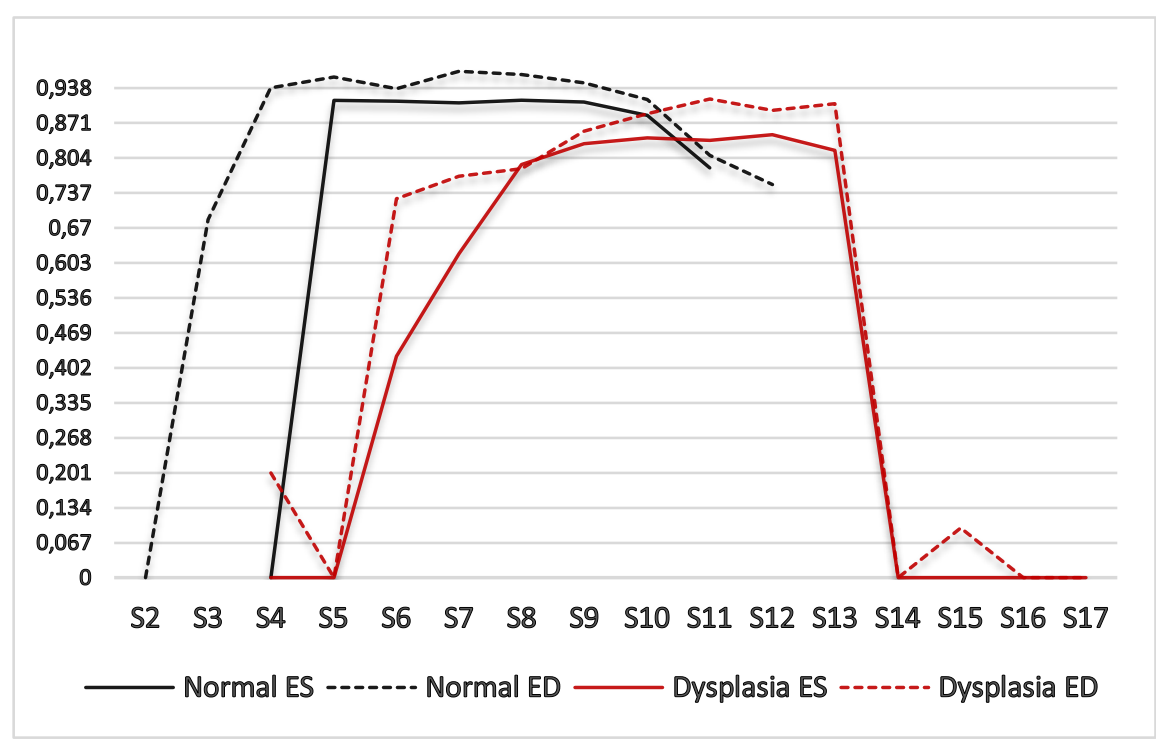

Fig. 4. Dice Score Metric evaluation of Short-Axis Slices from (S2 $\rightarrow$ S17) for both End-Systolic and End-Diastolic phases considering both normal and diseased patients 


\section{$5 \quad$ Conclusion and discussion}

In this paper, a deep-learning-based cardiac segmentation tool is considered for evaluating the impact of CMRI slice from base to the apex on the effectiveness of the segmentation. The metrical presented results in Fig. 3 and Fig. 4 show respectively the computed Accuracy and Dice metric values for each short-axis slice from base to apex (S2, S3...S17). The very first basal slice is excluded as it doesn't belong to the RV space. For the healthy patient, the included slices are S2, S3...S12. However, the pathological patient covers the whole 16 slices as the RV is dilated. For both patients, the very first basal and the very last epical slices associate low accuracy and DM. Besides, the accuracy and the DM results are low for the entire pathological patient' slices compared to the healthy slices. Thus, the segmentation results show that from central to basal and apical slices the effectiveness of the delineation decreases. Also, the case of dysplasia illustrates how widely the effectiveness decreases and how do the diseased cases influence the accuracy of segmentation. As a perspective, we attempt to tackle the basal and apical segmentation issues as well as the pathological problem toward a better effective segmentation process based on deep-learning approaches.

\section{References}

1. Sheehan, F., Redington, A.: The right ventricle: anatomy, physiology and clinical imaging. Heart. 94, 1510-1515 (2008). https://doi.org/10.1136/hrt.2007.132779.

2. Irshad, M., Sharif, M., Khan, M.Y. and A.: A Survey on Left Ventricle Segmentation Techniques in Cardiac Short Axis MRI, https://doi.org/10.2174/1573405613666170117124934, last accessed 2019/06/12.

3. Sami, S.M., Elfawal, S.K., Abdelgawad, M.S., Zidan, M.A., Zaki, A.M., Mowaki, A.F.: MDCT in the study of left ventricular function compared with MRI in patients with myocardial ischemia. Egypt. J. Radiol. Nucl. Med. 49, 29-41 (2018). https://doi.org/10.1016/j.ejrnm.2017.11.004.

4. Epstein, F.H.: MRI of left ventricular function. J. Nucl. Cardiol. Off. Publ. Am. Soc. Nucl. Cardiol. 14, 729-744 (2007). https://doi.org/10.1016/j.nuclcard.2007.07.006.

5. Petitjean, C., Zuluaga, M.A., Bai, W., Dacher, J.-N., Grosgeorge, D., Caudron, J., Ruan, S., Ayed, I.B., Cardoso, M.J., Chen, H.-C., Jimenez-Carretero, D., Ledesma-Carbayo, M.J., Davatzikos, C., Doshi, J., Erus, G., Maier, O.M.O., Nambakhsh, C.M.S., Ou, Y., Ourselin, S., Peng, C.-W., Peters, N.S., Peters, T.M., Rajchl, M., Rueckert, D., Santos, A., Shi, W., Wang, C.-W., Wang, H., Yuan, J.: Right ventricle segmentation from cardiac MRI: a collation study. Med. Image Anal. 19, 187-202 (2015). https://doi.org/10.1016/j.media.2014.10.004.

6. Cardiac MRI and CT Software - Circle Cardiovascular Imaging - Cardiac MRI, https://www.circlecvi.com/cvi42/cardiac-mri/, last accessed 2019/10/17.

7. Ou, Y., Doshi, J., Erus, G., Davatzikos, C.: Multi-Atlas Segmentation of the Cardiac MR Right Ventricle. Presented at the (2012).

8. Bai, W., Shi, W., Wang, H., Peters, N.S., Rueckert, D.: Multi-Atlas Based Segmentation with Local Label Fusion for Right Ventricle MR Images. Presented at the (2012). 
9. Sun, H., Frangi, A.F., Wang, H., Sukno, F.M., Tobon-Gomez, C., Yushkevich, P.A.: Automatic Cardiac MRI Segmentation Using a Biventricular Deformable Medial Model. In: Jiang, T., Navab, N., Pluim, J.P.W., and Viergever, M.A. (eds.) Medical Image Computing and Computer-Assisted Intervention - MICCAI 2010. pp. 468-475. Springer Berlin Heidelberg (2010).

10. Yang, X., Yeo, S.Y., Su, Y., Lim, C., Wan, M., Zhong, L., Tan, R.S.: Right Ventricle Segmentation by Temporal Information Constrained Gradient Vector Flow. In: 2013 IEEE International Conference on Systems, Man, and Cybernetics. pp. 2551-2555 (2013). https://doi.org/10.1109/SMC.2013.435.

- 11. Maier, O.M.O., Jiménez, D., Santos, A., Ledesma-Carbayo, M.J.: Segmentation of RV in 4D cardiac MR volumes using region-merging graph cuts. In: 2012 Computing in Cardiology. pp. 697-700 (2012).

12. Quispe, A.M., Petitjean, C.: Shape prior based image segmentation using manifold learning. In: 2015 International Conference on Image Processing Theory, Tools and Applications (IPTA). pp. 137-142 (2015). https://doi.org/10.1109/IPTA.2015.7367113.

13. Zotti, C., Luo, Z., Lalande, A., Jodoin, P.-M.: Convolutional Neural Network with Shape Prior Applied to Cardiac MRI Segmentation. IEEE J. Biomed. Health Inform. (2018). https://doi.org/10.1109/JBHI.2018.2865450.

14. Jang, Y., Hong, Y., Ha, S., Kim, S., Chang, H.-J.: Automatic Segmentation of LV and RV in Cardiac MRI. In: Pop, M., Sermesant, M., Jodoin, P.-M., Lalande, A., Zhuang, X., Yang, G., Young, A., and Bernard, O. (eds.) Statistical Atlases and Computational Models of the Heart. ACDC and MMWHS Challenges. pp. 161-169. Springer International Publishing (2018). 